\title{
COMENTARIOS SOBRE LA SITUACION ACTUAL DEL FUEGO Y SU RELACION CON LA MADERA
}

\author{
(COMMENTARIES ON FIRE IN SPAIN TODAY AND ITS RELATION TO WOOD)
}

Luis Miguel Elvira Martín, Dr. Ingeniero de Montes.

Jefe del Laboratorio del Fuego del INIA.

\section{RESUMEN}

Se expone brevemente la historia e intervención de los distintos Organismos en el estudio del fuego en nuestro país. Se definen conceptos fundamentales en este campo y su incidencia en el incendio, pasando revista al comportamiento de la madera en comparación con otros materiales. Junto con el análisis de la legislación y normalización, se termina exponiendo diversos problemas que se plantean en el campo del fuego al usuario y a la industria.
SUMMARY

The history and importance of various agencies connected with the study of fire in our country is briefly set forth Fundamental concepts in this field and their effect on fires are defined, while the behaviour of wood with respect to other materials is analyzed. After an analysis of the current legislative situation regarding fires, the article ends by setting forth various problems fire poses for consumers and industry.

\section{INTRODUCCION}

Los estudios del fuego y su relación con los problemas de la industria de la madera, asi como el análisis de la carga energética de los productos forestales y naturales, fueron preocupación del Departamento de Maderas del Instituto Nacional de Investigaciones Agrarias desde el año 1967 (Foto n. ${ }^{\circ}$ ).

Hacia 1976 el Laboratorio del Fuego creado en dicho departamento se integró en la labor de confección de

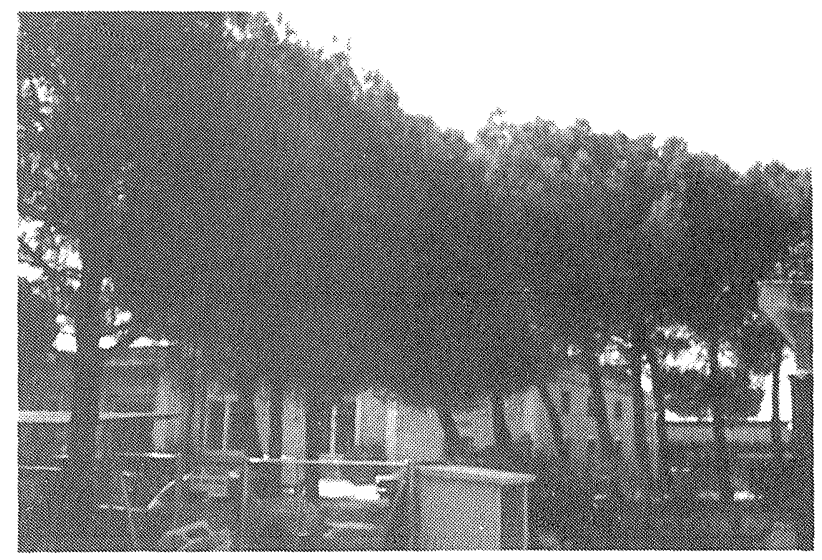

Foto 1.-Departamento de maderas del CIT (INIA). normas que iniciaba entonces BEQUINOR y en los estudios de la seguridad frente al fuego que también empezaba a patrocinar el Centro de Prevención de Daños y Pérdidas (CEPREVEN).

Los estudios del fuego, sin embargo, contaban ya con un período de experimentación de unos 30 años en paises de Europa.

Paralelamente, el Ministerio de Obras Públicas y Urbanismo iniciaba hacia 1974 sus primeros intentos de legislación del fuego en las edificaciones, mediante diversas Ordenes ministeriales, $y$ en este mismo año iniciaba también el Gobierno Civil de Barcelona su reglamentación sobre incendios.

Al amparo de esta preocupación inicial por el estudio del fuego surgieron nuevos laboratorios, que si bien nacian tímidamente equipados, ayudaron a configurar el esquema actual del fuego al concentrarse en IRANOR la labor de normalización de ensayos iniciada por BEQUINOR.

Ayuntamientos y Diputaciones, Ministerio del Interior, Ministerio de Comercio, etc., fueron integrándose posteriormente en las acciones a tomar frente al fuego. 
El panorama realmente disperso, que hacia 1981 presentaba el tratamiento de los incendios en las edificaciones, fue orientándose hacia un Ministerio (MOPU) que asumió la labor de legislación para las edificaciones no industriales, $M .^{\circ}$ de la Industria para estas últimas, apoyados por una red de laboratorios, centralización de normativa de ensayos (IRANOR-AENOR), entidades colaboradoras (AITIM, etc.) y Organismos encargados de la policía y vigilancia de cumplimiento de la reglamentación del fuego (Interior, Bomberos, etcétera).

No obstante la labor de concentración llevada a cabo desde entonces, consideramos que debe continuarse preparando la conjunción de esfuerzos y acciones frente al fuego, lo cual no debe impedir su proyección dentro de cada una de las actuales Autonomías, siempre que éstas sepan recoger la labor del conjunto.

Es frecuente, sin embargo, que el público en general (usuario de los productos, industrial, etc.) no tenga idea clara de las funciones que tiene cada uno de los entes citados y, por ello, a dónde debe acudir a la hora de utilizar o exigir una calidad frente al fuego de los materiales o estructuras.

Se precisa también facilitar una información, al menos elemental del comportamiento frente al fuego de aqueIlos materiales, para que puedan ser utilizados con idoneidad, así como establecer una idea clara del proceso de consecución de una calidad para los mismos y del uso correcto y prestaciones frente al fuego que el ciudadano puede esperar de tales materiales.

\section{CONCEPTOS BASICOS}

En el desarrollo del incendio en un edificio tiéne gran importancia el comportamiento de los materiales y las estructuras. El de los materiales en la primera fase del incendio y en la intermedia, y el de las estructuras en esta última y en la fase final.

Se tiene conocimiento del comportamiento al fuego de los materiales estudiando su reacción al fuego la cual mide el alimento que los mismos pueden aportar, es decir, el calor que generan, altura y virulencia de las Ilamas, toxicidad y opacidad del humo que desprenden, velocidad de avance de la llama, etcétera.

Respecto a la reacción al fuego los materiales se clasifican en cinco clases, desde M-1 (no inflamables) hasta M-5 (muy fácilmente inflamables), pasando por los grados intermedios.

Asi pues, el incendio de un edificio con materiales M-5 será espectacular, gran virulencia, grandes llamas y humo abundante. Es evidente que en este caso la actuación rápida de los bomberos es esencial en la primera fase del incendio para evitar la iniciación y desarrollo del mismo y la existencia de víctimas por intoxicación.

La la resistencia al fuego, por el contrario, mide el tiempo que una estructura (formada por diversos materiales con distintos grados de reacción) es capaz de soportar el incendio cumpliendo su misión en el edificio. Representa por tanto el tiempo de huida y salvamento de que se dispone ante el incendio.

El incendio de un edificio construido con materiales M-5 puede extinguirse, pues, fácilmente si la actuación de los bomberos es rápida, o detenerse si el edificio está compartimentado con tabiques resistentes al fuego, o no producirse derrumbamientos ni deterioros importantes o víctimas si sus estructuras tienen resistencia al fuego adecuadas.

De aquí se deduce que no puede decirse taxativamente que los materiales inflamables no puedan utilizarse en la construcción, o que sólo puedan emplearse estructuras altamente resistentes. Todo el conjunto del edificio debe tener una seguridad como consecuencia de la calidad ponderada frente al fuego de cada uno de sus materiales y estructuras.

No es correcto cargar las consecuencias de un incendio a un material ni a una estructura, sino a la defectuosa calidad frente al fuego o seguridad del conjunto.

Es frecuente escuchar, cuando se producen catástrofes por incendios, que han sido consecuencia de las estructuras de madera, o de las metálicas, o del material almacenado, etc. Lo primero es falso, se dice por desconocimiento, pues las de madera son las más resistentes al fuego; lo segundo incompleto, pues las metálicas debidamente protegidas pueden ofrecer una seguridad adecuada, y finalmente el material almacenado debe limitarse. En consecuencia, a veces no se dispone de las medidas de seguridad de conjunto que la reglamentación actual prescribre.

En el cuadro de la siguiente página se resumen los conceptos expuestos.

\section{COMPORTAMIENTO AL FUEGO DE LOS MATERIALES}

\section{Reacción al fuego}

- Es el alimento que un material puede aportar al fuego y al desarrollo del incendio, permitiendo apreciar el riesgo existente en el local, creador de pánico. Es, por tanto, un índice de la capacidad del material para favorecer el desarrollo del incendio. Este índice debe estar limitado en un potencial calorífico máximo admisible por $\mathrm{m}^{2}$ del local.

- En el caso de la madera se reduce mediante ignifugación de la misma. 


\section{I.N.I.A. \\ LABORATORIO DEL FUEGO}

\begin{tabular}{|c|c|c|c|c|c|c|}
\hline $\begin{array}{l}\text { RESISTENCIA } \\
\text { ALL } \\
\text { FUEGO } \\
\text { IIEMPO CUE UN } \\
\text { ELEMENTC REAL } \\
\text { PUEDE SCPORTAR } \\
\text { EL INCENDIO. } \\
\text { TIEMPO DE HUIDA } \\
\text { TIEMPO OE SALVAMENTO }\end{array}$ & $\begin{array}{l}\text { VARIABLES } \\
\text { ESTABILIDAD } \\
\text { MECANICA } \\
\text { ESTANQUEIDAD } \\
\text { DESPRENDIMIENTO } \\
\text { DE GASES INFLA- } \\
\text { MABLES. } \\
\text { AISLAMIENTO } \\
\text { TERMICO }\end{array}$ & $\begin{array}{l}\text { MATERIALES } \\
\text { CLASES. } \\
\text { ESTABLE AL } \\
\text { FUEGO } \\
\text { PARALLAMAS }\end{array}$ & $\begin{array}{l}\text { GENERALES } \\
\text { UNE- } 23.093 \\
\text { ISO } 834 . \\
\text { PUERTAS } \\
\text { UNE - 23.802 } \\
\text { ISO } 3.008\end{array}$ & $\begin{array}{l}\text { DE INVESTIGACIO } \\
\text { O DE CONTRAST } \\
\text { HORNO } \\
\text { REALES O DE } \\
\text { HOMOLOGACION } \\
\downarrow \\
\text { CON CARGA } \\
\text { SIN CARGA }\end{array}$ & $\begin{array}{l}\text { Y Y HORNOS } \\
\text { ONE } 90 \times 90 \mathrm{~cm} \text {. } \\
\text { ON } \\
\text { HORNO } \\
\text { VERTICAL } \\
\text { ELEMENTOS COLOCA. } \\
\text { DOS VERTICALMENTE } \\
\text { IPUERTAS, TABIQUES. } \\
\text { MUROS. POSTES.) } \\
\text { HORNO } \\
\text { HORIZONTAL } \\
\text { ELEMEMTOS COLO- } \\
\text { CADOS MORIZONTAL- } \\
\text { MENTEICUBIERTAS. } \\
\text { SUELOS.TECHOS } \\
\text { VIGASI }\end{array}$ & 7 \\
\hline
\end{tabular}

- Según este criterio se clasifican los materiales en las siguientes categorias:

\section{INCOMBUSTIBLES \\ $\mathrm{M}-0$}

\section{COMBUSTIBLES}

$M-1 \quad$ No inflamable

M-2 Difícilmente inflamables

M-3 Medianamente inflamables

M-4 Fácilmente inflamables

M-5 Muy fácilmente inflamables

\section{Resistencia al fuego}

- Tiempo durante el cual los elementos son capaces de cumplir su función al ser atacados por el fuego.

- Se consideran:

1 Estabilidad mecánica.

2 Estanquidad a las Ilamas.

3 No emisión de gases inflamables.

4 Aislamiento térmico.

- Según estas propiedades se clasifican los materiales en categorías, citando el tiempo transcurrido hasta que falla en una cualquiera de aquéllas. Las categorías más frecuentes son:

\section{ESTABLE AL FUEGO}

Se exige solamente la estabilidad mecánica (1).

PARALLAMAS

Se exige además la estanquidad a las llamas y no emisión de gases inflamables (1, 2 y 3 ).

\section{CORTAFUEGOS}

Se exigen las cuatro propiedades $(1,2,3$ y 4$)$.

\section{MEDIDA DE COMPORTAMIENTO AL FUEGO. ENSAYOS Y NORMAS}

Se puede conocer el comportamiento al fuego de tres formas distintas:

1. Ensayos en laboratorio o semirreales.

2. Ensayos reales.

3. Mediante cálculos.

Los ensayos reales no se utilizan en nuestro país. Solamente a efectos de investigación se llevan a cabo en algunos paises. Tienen lugar sobre edificaciones reales generalmente edificios pequeños, de una o dos habitaciones, incluso amuebladas, donde se mide el com- 
portamiento de materiales y estructuras en función de la temperatura y características del incendio interior. Su costo es elevado por ser ensayos en los que debe llegarse generalmente a la destrucción del edificio.

El conocimiento del comportamiento al fuego mediante cálculos tiene un campo muy limitado. Sólo se puede utilizar a efectos del conocimiento aproximado, para proyectos, diseños, etc. Unicamente por este procedimiento pueden obtenerse resistencias próximas a las reales obtenidas mediante ensayos, en estructuras de naturaleza uniforme y de construcción estandarizada tales como vigas o estructuras simples de acero, y no puede aplicarse a estructuras combustibles ni inflamia bles.

Dicho procedimiento se refiere únicamente al conocimiento de la resistencia al fuego y no al de reacción al fuego.

La resistencia al fuego de una estructura no depende únicamente de ella sino de su colocación y a veces de la de sus propios elementos. Esto tiene un peso muy importante en aquella resistencia, que podríamos $\mathrm{ci}$. frarlo en un $50 \%$ en algunas estructuras. En los procedimientos de cálculo no pueden considerarse estas circunstancias y de aquí nace la limitación del procedimiento.

Los ensayos del laboratorio proporcionan un conocimiento de la resistencia al fuego muy próximos a la realidad. Ello ha sido contrastado en numerosas ocasiones y por ello es por lo que se utiliza pra homologar, clasificar y dar una calidad frente al fuego a elementos y estructuras.

Los ensayos de reacción al fuego no son iguales en todos los países. No obstante se trabaja a efectos de unificarlos, si bien como diremos más adelante surgen dificultades que relentizan la solución de este problema. Nuestras normas de ensayo son muy parecidas a las utilizadas en Francia, país de buena tradición en la lucha contra el fuego.

Los ensayos de resistencia al fuego son prácticamente los mismos en todos los países europeos y difieren algo de los americanos. Se pretende mantener esta uniformidad de normativa en Europa, e incluso se van perfilando criterios de interpretación, etc., que pudieran llevar a clasificaciones distintas de una misma estructura en distintos países.

Así el esquema actual del estudio del fuego se aprecia, por lo que hemos apuntado anteriormente, la falta de dedicación de un laboratorio de ensayos al contraste de normas UNE, corrección de sus defectos, comprobación de reproductividad de dichas normas, aclaración de interpretaciones y utilización de tales normas. Ello haría posible la obtención de la misma clasificación para un material ensayado en distintos laboratorios del país.

Con el fin de no entrar en demasiados detalles sobre el tema señalado, en el presente Apartado, ofrecemos un resumen de las normas de ensayos utilizadas en nuestro país, pues recibimos frecuentemente peticiones de ensayos del fuego sin especificación alguna, tanto de la persona que los solicita (usuario) como de la autoridad que los exige (Administración). Esta circunstancia será comentada posteriormente en otro Apartado. En dicho resumen, se indica la correspondiente norma ISO con la que existe analogía, y a veces la clasificación del material que permite dicha norma, o las variables que mide y se ofrece un esquema del horno o aparato utilizado.

\section{MATERIALES Y ESTRUCTURAS}

No debe creerse que el incendio es un accidente moderno. Grandes incendios ocurrieron en la Edad Media, que asolaron conjuntos enteros de edificaciones; edificaciones con madera, material abundante utilizado entonces para la construcción, que al estar defectuosamente colocado y utilizado, ha asumido tradicionalmente la fama de pasto fácil para el incendio.

Posteriormente, y en parte creyendo solucionar entre otros este problema, se empleó el acero, como material constructivo, comprobándose actualmente que también presenta frente al fuego determinados problemas.

El hormigón, que en cierta forma enmascara dicha respuesta defectuosa del acero, presenta sin embargo otras peculiaridades no deseables en su comportamiento al fuego.

De todo ello hablaremos seguidamente para llegar a la conclusión de que la construcción puede y debe contar con la amplia gama de materiales que la tecnología ofrece, desde la madera hasta el hormigón, jugando con ellos dentro de la economía o estética deseables para el edificio, sin prescindir de ofrecer la seguridad frente al fuego que se desee. Unicamente es preciso disponer y utilizar correctamente estos materiales.

\subsection{La madera en comparación con otros materiales}

Observamos edificaciones antiguas, aún en pie en el centro de las grandes ciudades, construidas con estructura de madera, cerrada con mampostería incluso de adobe, que a través de la tomiza o cuerda de esparto liada (con funciones de adherencia) sobre la madera, hace de mecha que transmite el fuego de una pieza a otra de la estructura de madera. Esta madera, sin aireación, se encuentra a veces reseca, a veces ataca- 
da por insectos, por no haber sido tratada con polvo en la superficie procedente de las galerias de tales insectos, y por tanto en condiciones ideales para arder; tales galerias actúan de chimeneas y favorecen la combustión. La resistencia mecánica de las vigas se encuentra disminuida por tales perforaciones, que a veces reducen las piezas a su estructura elemental leñosa. Pues bien, a pesar de todo ello, cuando se produce un incendio en edificios de este tipo, los bomberos se fían de la estructura porque saben que permanece en pie aún después del incendio, permitiendo la huida y salvamento (Foto n. 2 ).

Difícilmente podremos encontrar un incendio en edificios que tengan algunos años, donde no intervenga la madera, y de todos aquellos en los que esté representada se dirá que este material fue causa del siniestro, o porque ardieron escaleras de madera o las vigas de su estructura o la madera que recubría las paredes. El lector lo comprenderá fácilmente y quedará en ese sentido resignado haciendo las correspondientes lamentaciónes.

Sin embargo, no es frecuente que se mencione, porque no es tan llamativo y exige algunas reflexiones: que la madera difícilmente puede ser la causa, aunque sí el alimento del incendio; que después del mismo el edificio quedó en condiciones aceptables y que los bomberos pudieron actuar con seguridad ante la firmeza de sus estructuras, salvando posibles víctimas y enseres.

Por el contrario, comparando este tipo de incendios con los que ocurren en edificios modernos, donde la estructura no es de madera, o existen moquetas, telas o plásticos, se observan en éstos, por lo general, derrumbamiento casi total de la estructura, vícimas por asfixia o envenenamiento, dificultad en el salvamento, resbalamiento en escaleras durante la evacuación por f'uidificación de los plásticos, gran carga calorífica, etc. En tales casos, al no poderse atribuir la causa a la ma-

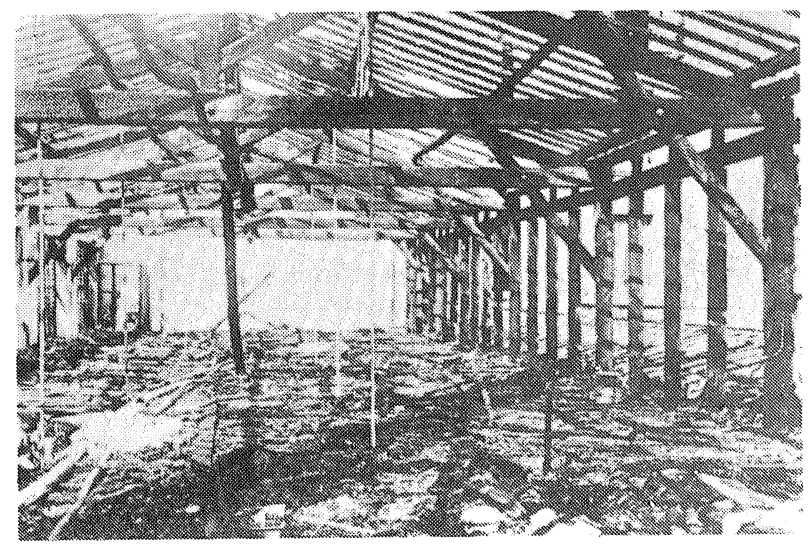

Foto 2.-Estructura de madera en pie después del incendio. dera, se menciona al propietario, a la falta de medidas de seguridad o su incumplimiento o, finalmente al azar; se propaga la sicosis del fuego y se arbitran medidas urgentes y acciones inmediatas, que por ser precipitadas suelen ser poco efectivas, si bien es justo reconocerlo sirven de base para posteriores correcciones en cuanto a seguridad. Todo ello confirma lo expuesto al principio de este Apartado 4.

A consecuencia del empleo de los materiales modernos se iniciaron los estudios del fuego, pero no al de la madera, de la que se supo poco en cuanto al fuego, y por ello se consideró siempre como material peligroso. Su estudio, surgido con posterioridad a los incendios espectaculares modernos de las materias sintéticas, ha revelado que la madera posee una alta resistencia al fuego y buenas posibilidades de reducir su reacción.

Ello explica el que el estudio del fuego se haya iniciado en época reciente, en que la madera, por su escasez, ha cubierto otros campos como el de la fabricación de pastas, mediante turnos cortos de explotación, dejando sin atender a la construcción, al menos en el aspecto estructural que requería grandes escuadrías. Resuelto hoy este vacio mediante las estructuras laminadas y la excelente calidad de los tableros es preciso considerar el comportamiento de la madera ante el fuego en comparación con otros materiales y en su verdadera dimensión.

\subsubsection{Reacción al fuego}

1. La madera gruesa arde difícilmente y si no se aporta calor externo se apaga. Las vigas y estructuras de los edificios sólo arden superficialmente (Foto n. ${ }^{\circ}$ 2). El carbón formado en la capa exterior impide el paso de oxígeno al interior de la viga y la combustión tiende a frenarse. El paso de calor al interior de la misma es cada vez más difícil dado el bajo coeficiente de transmisión de calor de este ma-

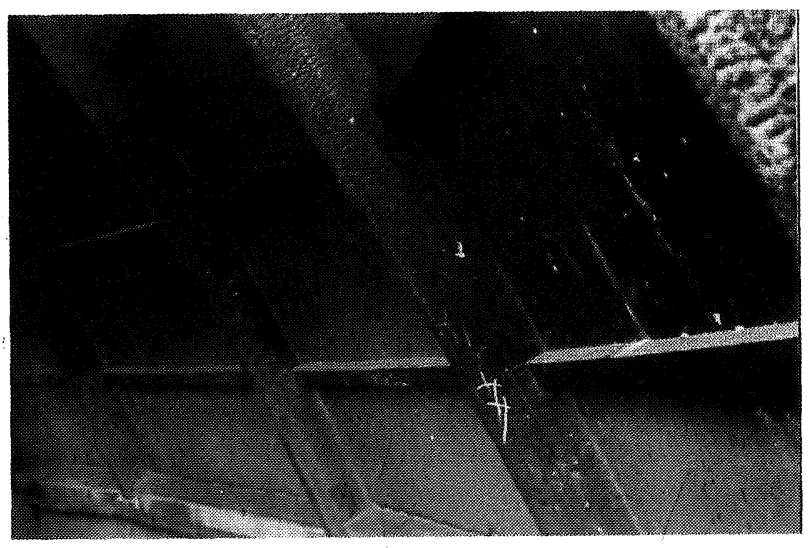

Foto 3.-Carbonización e inflamación superficial de las piezas gruesas de madera. 
terial. En general las estructuras de madera permanecen en pie después del incendio o si se derrumban lo hacen parcialmente. De aquí que las vigas no requieran ignifugación en general, o si acaso superficial. En profundidad únicamente en vigas antiguas y secas. Se precisa sin embargo un tratamiento contra insectos y hongos (Foto n. ${ }^{\circ} 3$ ).

2. Si el material es de poco espesor y no se encuentra debidamente colocado la trasmisión del fuego se efectúa con facilidad. Es preciso conocer y prevenir en la intervaloración fuego-madera, la colocación de ésta en el edificio, su distribución sobre todo en las piezas de poco espesor, chapas, tableros contrachapados y material de recubrimiento y decorativo. Evitar zonas próximas con cargas importantes de fuego. Los paramentos verticales, donde la velocidad de avance del fuego es alta, deben ignifugarse. La colocación de telas o chapas de madera como revestimientos apoyados en rastreles con cámara hasta el muro o tabique suele ser actualmente frecuente y como vemos peligrosos. Deben cuidarse igualmente las superficies libres terminadas en aristas y ángulos vivos.

3. En un edificio existen materiales que hacen el efecto de fulminante; lo representan las cortinas o telas, papeles, muebles, moquetas, etc. que sí son la causa inmediata del fuego y no la madera, sobre los cuales incide inicialmente al foco de calor.

Unicamente asume este papel la madera cuando está excesivamente seca y en forma de polvo: vigas carcomidas, serrín, etc. En estos casos pues, debe alejarse de los focos de calor.

4. Es imposible que la madera arda mientras contenga humedad. Debe colocarse en zonas abiertas para que esté suficientemente húmeda y en equilibrio atmosférico asegurando una protección inicial. EI agua que posee absorbe calor para evaporarse $y$, mientras existe, la madera no arde ganándose tiem. po frente al incendio.

5. La potencia del foco calorífico también es importante a la hora de disponer los materiales. Así un parquet no puede entrar en combustión mediante una cerilla, etc. Pocas veces en la propia madera se inicia el incendio. Podrá permitirse o no su presencia en el foco según la potencia calorífica de éste.

6. La utilización de maderas de especies poco inflamables y altamente resistentes al fuego debe establecerse en zonas de posible peligro.

Todas estas circunstancias a tener en cuenta en la combustión y dinámica del fuego en la madera se conocen mediante estudios de investigación desarrollados en el INIA. A conclusiones análogas se ha llegado en otros países donde la madera se emplea ampliamente en la construcción.

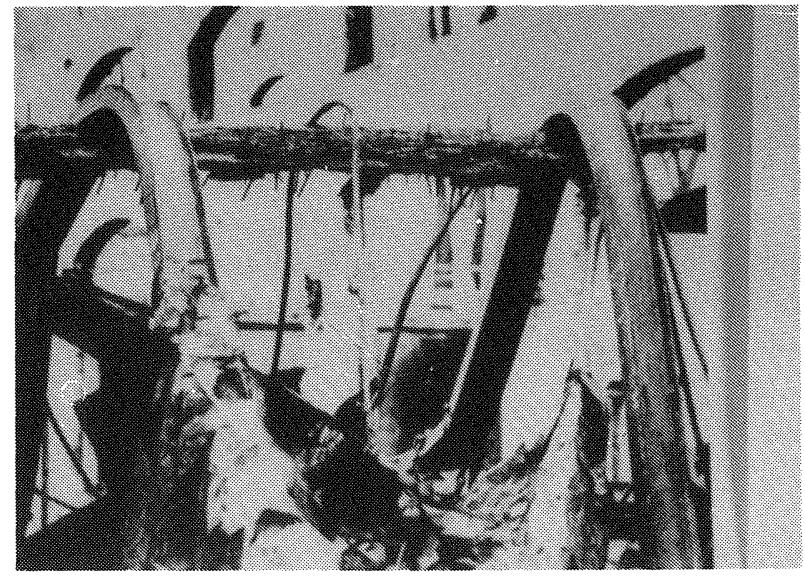

Foto 4.-Diferencia entre la madera y los metales en cuanto a su re sistencia al fuego.

Venimos por tanto diciendo que la madera arde, pero esta dificultad de tener alta reación al fuego puede y debe corregirse mediante ignifugación y uso adecuado. En general se admite que el límite entre las clases M-4 (fácilmente inflamables) y M-3 (medianamente inflamables) en resinosas son los $18 \mathrm{mi}$ límetros de espesor; dicho límite en frondosas desciende a $14 \mathrm{~mm}$. Tiene sin embargo una alta resistencia al fuego se hace patente especialmente en las piezas más gruesas, es decir tiene la facultad de soportar o aguantar al incendio durante mucho tiempo, obteniéndose como cifra media al paso de fuego de $0,7 \mathrm{~mm} / \mathrm{min}$. Ocurre lo contrario con el hierro y metales, en los que la llama se desarrolla con dificultad, pero transmiten fácilmente el calor y se dilatan y deforman, arruinándose las estructuras (Foto $\left.n .^{\circ} 4\right)$.

En cuanto a éstas, cuando se trata de madera, poco o muy poco problema plantean frente al fuego, pues ya hemos hablado del comportamiento de piezas gruesas, pero además en el caso de la madera laminada, al estar encolada generalmente con colas termoendurecibles, disfruta de una alta resistencia sin necesidad de tratamiento alguno.

\section{Ignifugación}

La madera es combustible e inflamable en estado natural, pero puede eliminarse esta inflamabilidad totalmente y en parte la incombustibilidad, mediante tratamientos ingnífugos.

Existen productos que por fundir a baja temperatura rellenan los poros de la madera impidiendo la salida de gases inflamables.

Otros forman espumas o barreras logrando el mis. mo efecto. Por el contrario, ciertos productos ignífugos permiten la salida normal de gases inflamables, pero emiten bajo la acción del fuego gases no inflamables que al mezclarse con los primeros y rebajar su proporción en el aire, retardan la velocidad de inflamación. 
Otro tipo de ignífugos actúan directamente sobre el proceso de combustión rompiendo las reacciones que en ella tienen lugar.

Ya hemos visto antes que al ser atacada la madera por el fuego en la primera fase, hasta que adquiere los $100^{\circ} \mathrm{C}$ se produce el secado, retardando el incendio. La ignifugación aprovecha este efecto utilizando productos que humedecen la madera, bien sea tomando el agua de la atmósfera o a partir del agua cristalizada del propio producto.

Otros, finalmente son sales que forman con la madera compuestos orgánicos más estables, o bien, como en el caso de los fosfatos, pueden aumentar hasta un $60 \%$ la cantidad de carbón y la de vapor de agua, reduciendo la incandescencia del carbón, con lo que se evita el desprendimiento de gases inflamables.

Estas diversas formas de actuación de los productos ignífugos pueden presentarse combinadas a la vez en algunos de ellos lográndose, según la técnica de aplicación y la intensidad, el grado de protección que se desee. Ello es en definitiva un problema puramente económico a considerar por el fabricante, según las condiciones del mercado.

\subsubsection{Resistencia al fuego.}

Para medir la resistencia al fuego de un elemento se le somete a las temperaturas simuladas del incendio según la curva:

$$
\triangle T=K \log (8 t+1)
$$

siendo $\mathrm{K}$ una constante que para cada caso es función de carga combustible del edificio.

Se miden las siguientes propiedades o criterios mediante normas propias de cada pais, si bien se derivan en casi todos ellos de la ISO-DIS-834.

- Estabilidad mecánica, bajo su carga normal.

- Estanquidad a las llamas, tiempo que tardan en formarse grietas o perder su integridad dejando pasar las llamas.

- No emisión de gases inflamables.

- Aislamiento térmico, es decir, el paso del calor a través del elemento.

El tiempo que transcurre hasta que falla alguno de estos criterios, mide la resistencia al fuego del elemento.

Mientras mantiene las exigencias establecidas para los cuatro criterios, el elemento es CORTAFUEGOS y se dirá cortafuegos $1 \mathrm{~h}, 2 \mathrm{~h}$, etc., hasta que falle en alguno de ellos.

Si falla en el aislamiento térmico y sigue satisfaciendo los otros tres criterios, el elemento es PARALLAMAS (durante ese tiempo). Un elemento podrá ser, pues, $2 \mathrm{~h}$ cortafuegos y $5 \mathrm{~h}$ parallamas.
Si se exige o satisface solamente el primer criterio, el elemento es ESTABLE AL FUEGO.

Podrá clasificarse pues un mismo elemento como $2 \mathrm{~h}$ cortafuego, 3h parallamas y $4 \mathrm{~h}$ estable al fuego.

La madera sin ignifugar hemos visto que tiene mala reacción al fuego, aunque los ignifugantes la reducen. Sin embargo, tiene una alta resistencia al fuego pudiendo contener el incendio y mantener en pie las partes del edificio mientras se desaloja.

En efecto, la carbonización exterior de la madera y su baja conductibilidad térmica no permiten arder a la parte interna de las vigas y piezas grandes. La estructura al quedar carbonizada pierde su resistencia por la disminución del diámetro, pero es capaz aun de mantener su integridad.

Ocurre lo contrario con el hierro y el hormigón, buenos materiales en cuanto a su reacción al fuego y malos en cuanto a su resistencia al fuego. Los incendios en edificios construidos con estos materiales eran antes menos aparatosos, producían menos pánico (no tanto hoy dia por la carga combustible que suelen contener cortinas, moquetas, plásticos y fibras en general), pero dejan de cumplir su función antes que la madera y las construcciónes se derrumban en el fuego total e inesperadamente. El hormigón y sobre todo el acero deben protegerse respecto al fuego.

Como regla general se puede decir que la madera se comporta mejor que el hormigón, acero y aluminio. En efecto, estos dos últimos pierden resistencia y rigidez con el calor, mientras que la madera mala conductora del calor, tarda más en calentarse por una parte, y por otra, al deshidratarse, aumenta su resistencia a la compresión en un $4 \%$ y a la flexión en un $2 \%$ por cada $1 \%$ de decrecimiento de humedad. Ello compensa en exceso a las pérdidas de resistencia por disminución de la sección al arder.

En definitiva, y a título de ejemplo, puede admitirse que una viga de acero de $12 \mathrm{~m}$ a los tres minutos de estar sometida al incendio tiene un límite elástico inferior al admisible, mientras que la madera no se ha inflamado aún. El límite de resistencia de aquélla es inferior al admisible a los 7 min y sometida a $500^{\circ} \mathrm{C}$ se alarga a $72 \mathrm{~mm}$, lo cual es suficiente para producir el pandeo o desplazar los apoyos, provocando el hundimiento de la estructura. Esto en cuanto se refiere a estabilidad mecánica. Ello no quiere decir que no pueda emplearse debidamente protegido.

La estanquidad a las Ilamas presenta una gran variabilidad, según los materiales de que se trate, incluso en la madera, ofreciendo valores muy altos en algunas maderas sin ignifugar.

El fallo de la resistencia al fuego por estanquidad de los elementos de madera, se pone de manifiesto en aquellas estructuras de cierre de huecos (puertas, ven- 


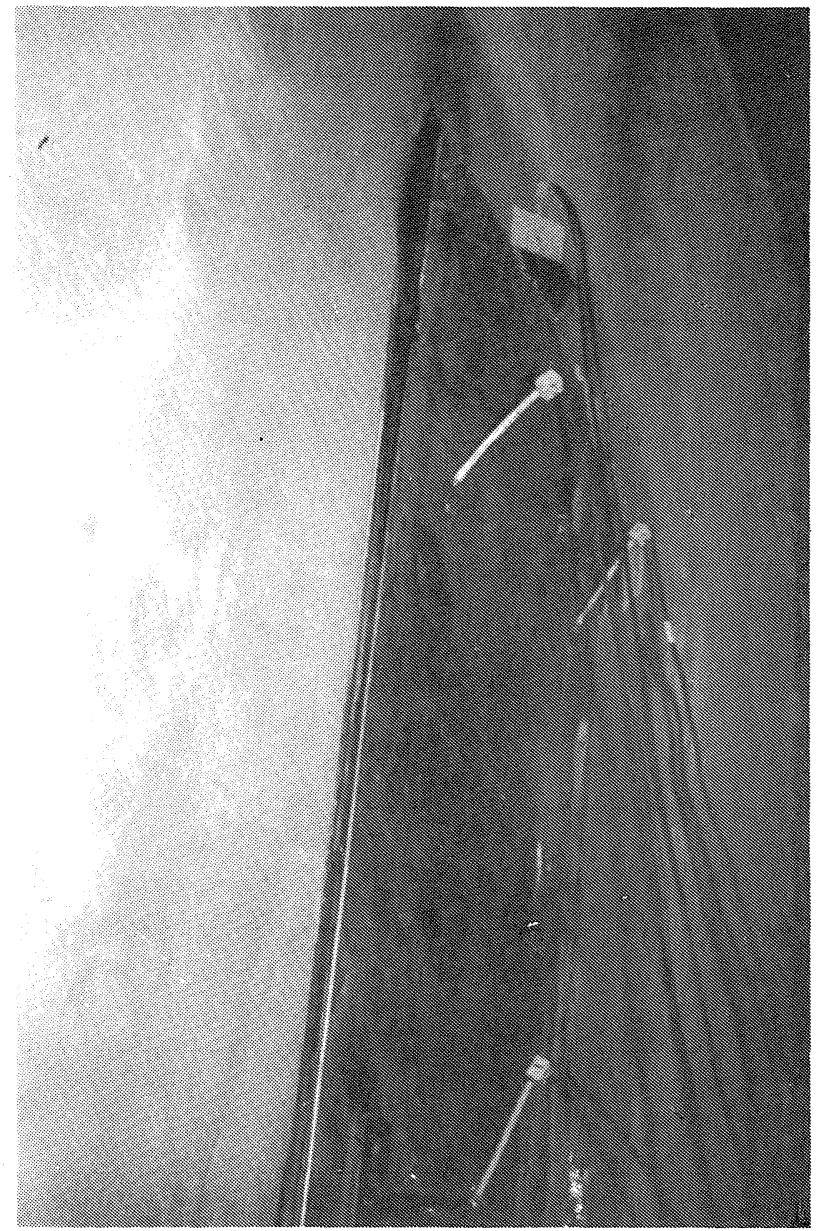

Foto 5.-Dilataciones en una puerta metálica.

tanas, etc.) Se admite que la velocidad de penetración del fuego en la madera es de $0,7 \mathrm{~mm} / \mathrm{min}$, despreciando los 3 primeros $\mathrm{mm}$ de formación de carbón.

Aunque la madera hemos visto que emite gases inflamables (hidrocarburos), lo hace por la cara expuesta, ya que la transmisión de calor a la cara externa es muy débil. No suele, por tanto, observarse este defecto. En el caso del aislamiento térmico al ser baja la conductibilidad térmica, el fuego en la cara expuesta no suele ser capaz de calentar la otra cara. Se tiene, pués, un excelente aislante que facilita la lucha contra el incendio.

Merece especial mención el caso de las puertas. Se cree generalmente que una puerta metálica especialmente reforzada, es muy resistente al fuego. Este tipo de puertas puede ofrecer una alta seguridad frente a robos, pero muy poca frente al incendio. Cuanto mayor sea la masa metálica, mayores son las dilataciones, produciéndose antes las holguras con el marco a través de las cuales pasará la llama. Igualmente la cara no expuesta al fuego se calentará rápidamente por conducción fácil del calor a través del metal, sobrepasando el límite admisible. Los refuerzos son perjudiciales, pues conducen a dilataciones imprevisibles, pudiéndose sumar sus efectos. Cuantos más puntos de cie-

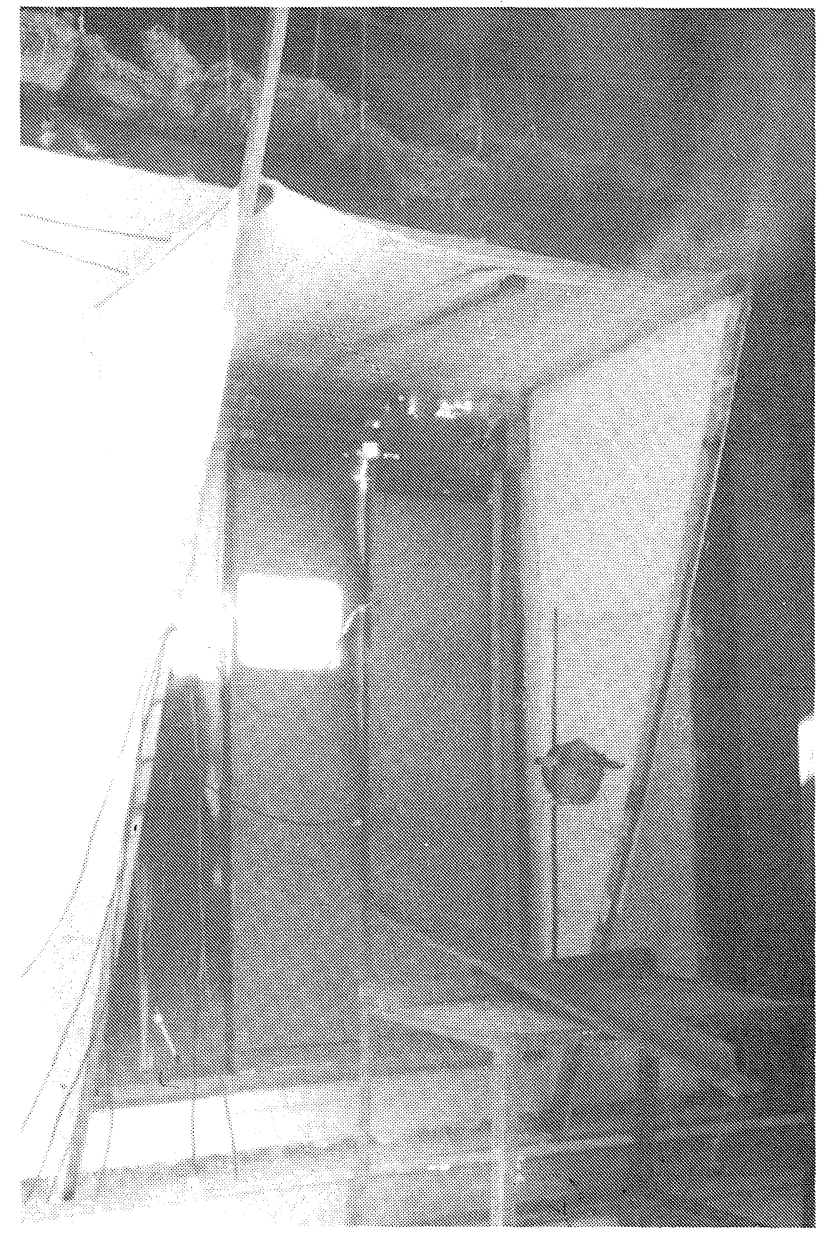

Foto 6.-Puerta metálica de ascensor. Doble hoja.

rre tenga la puerta mayor será la rigidez del conjunto, y al no poder dilatar libremente las partes, se deforma dicho conjunto (Fotos . $^{\circ} 5$ y 6 ).

Las puertas metálicas, para ser resistentes al fuego, necesitan tener un cuidadoso diseño, lo más ligero posible, $\sin$ refuerzos o en posiciones estudiadas, aisladas en sus caras y sin puentes térmicos, con puntos de cierre aislados de las superficies y con discontinuidad respecto a la transmisión del calor. Es suficiente con que un picaporte transmita el calor desde la cara expuesta al fuego a la no expuesta, para dar por terminado el ensayo. Ni la hoja ni el marco pueden sufrir dilataciones excesivas o desquilibradas, pues al final del ensayo tanto la cerradura como la hoja deben abrirse. Todo ello hace que la construcción de una puerta metálica resistente al fuego requiera especial cuidado en su construcción y montaje. Como consecuencia tendrá un costo elevado.

Las puertas de madera resistentes al fuego tienen un coste muy bajo, pues basta un simple tablero de partículas, incluso sin ignifugar, para dar a la hoja una alta resistencia al fuego, tanta como queramos sin más que aumentar su espesor, cuidando los cierres u holguras con elementos intumescentes y aislar los herrajes respecto a la madera. No existen prácticamente dilatacio- 


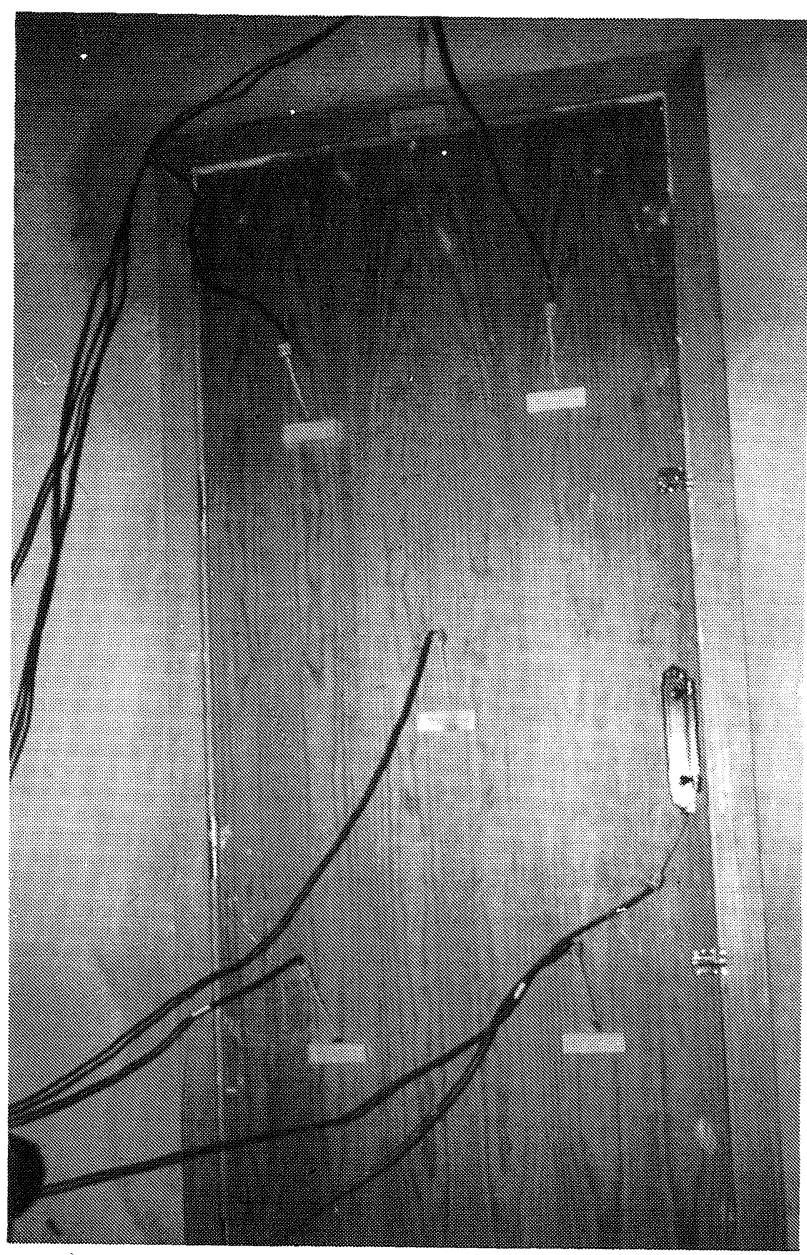

Foto 7.-Puerta de madera cortafuegos hora y media.

nes y la transmisión del calor de una cara a otra es muy lenta. Ello ha dado al mercado puertas cortafuegos de $1 / 2,1$ y $1 \frac{1}{2}$ horas a precios muy reducidos, y con el correspondiente sello de calidad frente al fuego (Foto n. $\left.{ }^{\circ} 7\right)$.

Si las puertas de madera son elementos clave en la lucha contra el incendio, no lo son menos las estructuras para edificar y especialmente las de madera laminada. Estas se construyen con colas termoendureci-

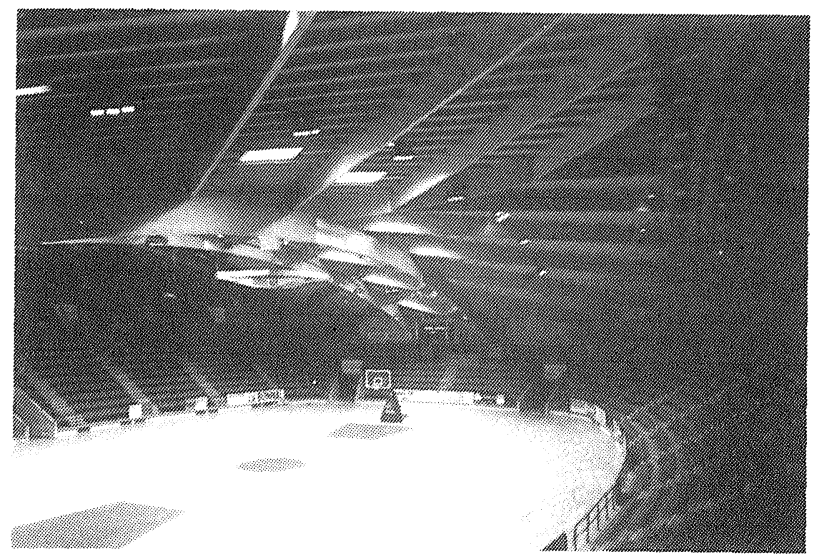

Foto 8.-Estructura de madera laminada. bles (resorcina) que no se inflaman con el calor, por lo que dichas vigas o postes no arden con el fuego más que muy difícil y superficialmente, característica que también poseen (aunque en menor grado) las vigas de madera maciza, si bien en este caso (como veíamos al principio) se precisa tratarlas no contra el fuego, sino contra hongos e insectos, para prevenir la transmisión de la llama cuando el edificio sea viejo.

Por su gran belleza y único material con posibilidad de sustituir al acero y al hormigón, cuando se trata de cubrir grandes luces, debe considerarse la madera laminada idónea para estructuras, ofreciendo alta calidad frente al fuego (Foto n. ${ }^{\circ} 8$ ).

\section{LABORATORIOS}

\subsection{Esquema general}

Dentro de la red de laboratorios de ensayos existentes, cabe distinguir dos grupos:

\section{Laboratorios homologados por el MOPU.}

2. Laboratorios no homologados por dicho Organismo.

En ambos grupos existen laboratorios de reacción o de resistencia al fuego, o bien otros que pueden efectuar ensayos de las dos modalidades a la vez. Asimismo los ensayos de resistencia al fuego pueden efectuarse sobre estructuras que en el edificio no soportan carga alguna (como tabiques o puertas) o bien sobre los que sí están sometidos a carga (vigas, muros, etc.) Ello lleva a la clasificación de los laboratorios homologados en los siguientes tipos según Orden del MOPU de 4-2-85 (BOE n. 85 de 9-4-85) que amplía la Clase D-Fuego establecida a su vez en el Real Decreto 1565/84 (BOE n. ${ }^{0} 127$ de 10-9-84):

Subclase D1: Laboratorios de reacción al fuego.

Subclase D2: Laboratorios de resistencia al fuego sin carga.

Subclase D3: Laboratorios de resistencia al fuego con carga.

La emisión de un certificado de ensayo, o informe, por un laboratorio homologado, supone la aceptación del mismo por el MOPU a efectos de cumplimentación de la legislación vigente (Norma Básica de la Edificación CPI-82). Sin embargo, a efectos de experimentación, conocimiento de calidad o uso propio de la industria, es posible la ejecución de ensayos en laboratorios no homologados, en aquellos casos en que no se precise de una inclusión posterior de la calidad obtenida del producto o estructura dentro de la legislación vigente. Se deduce de ello, que los laboratorios tienen la misión solamente de ensayar, no de homologar, ni por supuesto de decidir si la calidad está de acuerdo con la exigida por la legislación. 
La relación inicial de laboratorios homologados apareció en la Orden de 14-10-82 (BOE n. 279 de 20-11-82) y aparecen homologados para ensayos de la NBE en la citada del 4-2-85. Dicha relación puede ser ampliada teniendo conocimiento de ella el MOPU que es el Organismo que otorga dicha homologación.

Mediante los ensayos efectuados dentro del grupo de laboratorios homologados, por las condiciones que se exigen a los mismos para tal homologación, puede tenerse una mayor garantía de su perfecta ejecución e incluso de la coincidencia de las clasificaciones finales de un mismo producto en distintos laboratorios.

Pero obsérvese que no aseguramos tal coincidencia, puesto que a pesar de efectuarse los ensayos mediante normas determinadas, existe variación en aparatos, utilización de éstos, interpretación de normas, competencia técnica del personal y otras circunstancias; circunstancias estas difíciles de controlar y que entran dentro de la actuación de cualquier profesional.

A estos efectos de unificación y garantía hacia el consumidor, se ha creado en nuestro país una Subcomisión de Laboratorios, dentro de la Comisión Permanente del MOPU, encargada de coordinar y unificar el trabajo de los laboratorios.

El mismo problema existe a nivel internacional, por lo que algunos laboratorios, cuya calidad es satisfactoria, están integrados en el grupo EGOLF (European Group of Official Laboratories for Fire-Testing), formado por los de la CEE que han sido admitidos en dicho grupo.

La Subcomisión de Laboratorios citada está relacionada con dicho Grupo EGOLF a fin de defender nuestros laboratorios integrados en la CEE y recoger las conclusiones de dicho grupo que pueden ser interesantes para nuestro país.

Se aprecia sin embargo la falta de actuación en nuestro país de un laboratorio debidamente equipado, que sirva de contraste al resto de los otros laboratorios a efectos de homologaciones.

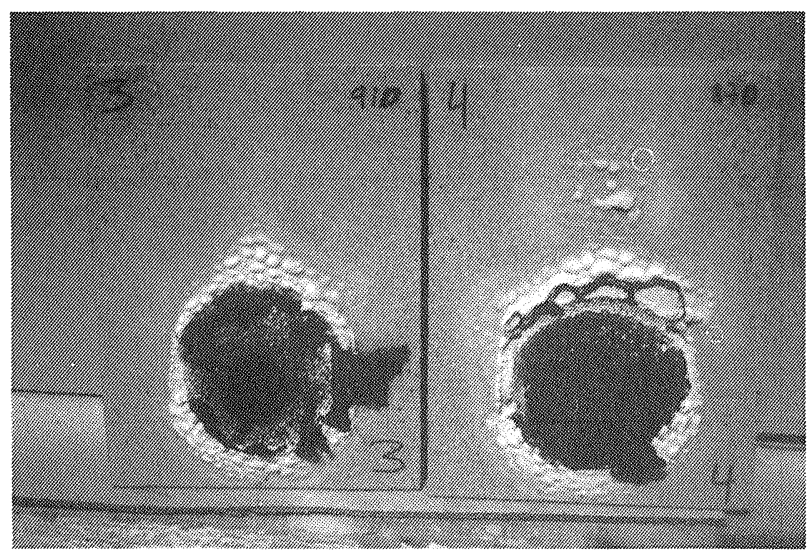

Tablero de particulas de madera no inflamable (M-1).

\section{LEGISLACION}

La legislación actualmente vigente está recogida en la Norma Básica de la Edificación NBE-CPI-82 -Condiciones de Protección contra Incendios de los Edificios.

Creada por Real Decreto 2059/81 de 10 de abril (BOE 18-7-81 y 19-7-81), corregida en el BOE 6-11-81 y modificada por Real Decreto $1587 / 82$ de 25 de junio (BOE de 21-7-82), ha sufrido posteriores modificaciones (BOE $n$. $^{\circ}$ 231 de 27-9-82) que la han dejado vigente solamente en su parte general, pero no en los Anexos.

Recogiendo la experiencia de dicha norma, la Comisión Interministerial Permanente que se crea en el último Real Decreto citado está redactando una nueva norma que sustituirá a la anterior. Es posible que dicha norma faculte al M. ${ }^{\circ}$ de Industria para regular dentro del ámbito de ella las actividades industriales.

Al amparo de la NBE-CPI-82 han surgido diversas reglamentaciones en las diferentes Autonomías que regulan la protección contra incendios en ellas, si bien tales reglamentaciones deben estar dentro de lo especificado por la NBE-CPI-82 de ámbito nacional.

No obstante, el Real Decreto de creación faculta al MOPU para ejercer las labores de vigilancia y cumplimiento de la norma general. Sin embargo, hay que citar el Reglamento General de Policía de Espectáculos Públicos y Actividades Recreativas del M. ${ }^{\circ}$ del Interior, Real Decreto 2816/82 de 27-8-82 (BOE n. ${ }^{\circ} 267$, de 6-11-82 y BOE 29-11-82).

\section{HOMOLOGACIONES, CERTIFICACIONES Y CONFECCION DE NORMAS. GARANTIA DE CALIDAD.}

La homologación es el reconocimiento oficial de un producto, proceso o servicio realizado por un Organismo que tiene esta facultad por disposición reglamentaria. En lo que se refiere a la NBE-CPI-82, actualmente vigente es, según esta definición, el MOPU el que dispone de esta facultad, tal como establece la reglamentación citada que crea dicha norma (Real Decreto 2059/81 de 10 de abril).

Según esto, entendemos que el MOPU es el eslabón final que garantiza la calidad de comportamiento al fuego de un material o estructura, a la vista de los informes emitidos por los laboratorios homologados por dicho Organismo.

Puede delegar dicha labor de homologación en otras Entidades que, con su aprobación, ayudarían a llevarla a cabo. En este sentido hay que citar a la Asociación 
de Investigación Técnica de la Industria de la Madera y Corcho (AITIM) que emite sellos y marcas de calidad respecto al fuego, para las estructuras fabricadas con madera. La utilización de una estructura con sello de calidad AITIM respecto al fuego supone notables ventajas. Una de ellas es la de poder disponer de otra estructura nueva y montada en caso de que la primera resultase falta de la calidad que garantiza su sello. Es decir, que si una puerta cortafuegos instalada en obra no proporciona la resistencia al fuego que marca su seIlo, el usuario puede reclamar otra nueva que ofrezca dicha resistencia, sin gasto alguno, incluso para su instalación. Los gastos correspondientes correrán a cuenta de AITIM, que a su vez los exigirá del fabricante.

En definitiva este es pues un proceso de vigilancia que la Administración puede ejercer sin movilizar grandes equipos de personal, mediante el propio control de la industria, puesto que las sanciones impuestas a la misma por incumplimiento de la calidad obligan a ésta a autocontrolarse por razones de competencia.

Como vemos, en el caso de la madera, sus derivados y estructuras está cubierto, con las debidas garantías, el proceso de calidad respecto al fuego desde su entrada al laboratorio de ensayos, hasta su utilización por el ciudadano. No conocemos que exista un proceso similar para las estructuras metálicas o de otros materiales al menos con un funcionamiento efectivo. Sin embargo ello es absolutamente necesario para completar el proceso de garantía de la calidad. De poco sirve que una estructura o material disponga de un certificado de ensayos, si por ejemplo se instala defectuosamente o se sustituye por otra a la hora de disponerlo en obra.

Para completar este Apartado debemos citar la reciente publicación del Real Decreto 16/4/85 de 1-8-85 (BOE n. ${ }^{\circ}$ 219 del 12-9-85) y posteriores disposiciones que le desarrollan, en el que se faculta al M. ${ }^{\circ}$ de la Industria y Energía para llevar a cabo funciones de certificación y normalización.

Mediante el mismo, este Ministerio controlará los procesos de certificaciones y normalización. En cuanto a este último, asume la confección de normas del fuego, en unión de AENOR, desapareciendo IRANOR y por lo tanto varía poco este aspecto de normativa dentro del panorama general del fuego. Puede no ser asi en cuanto se refiere a las certificaciones, respecto a las cuales es preciso esperar a que se desarrolle en toda su amplitud el citado Real Decreto, que puede incidir en competencias y funciones.

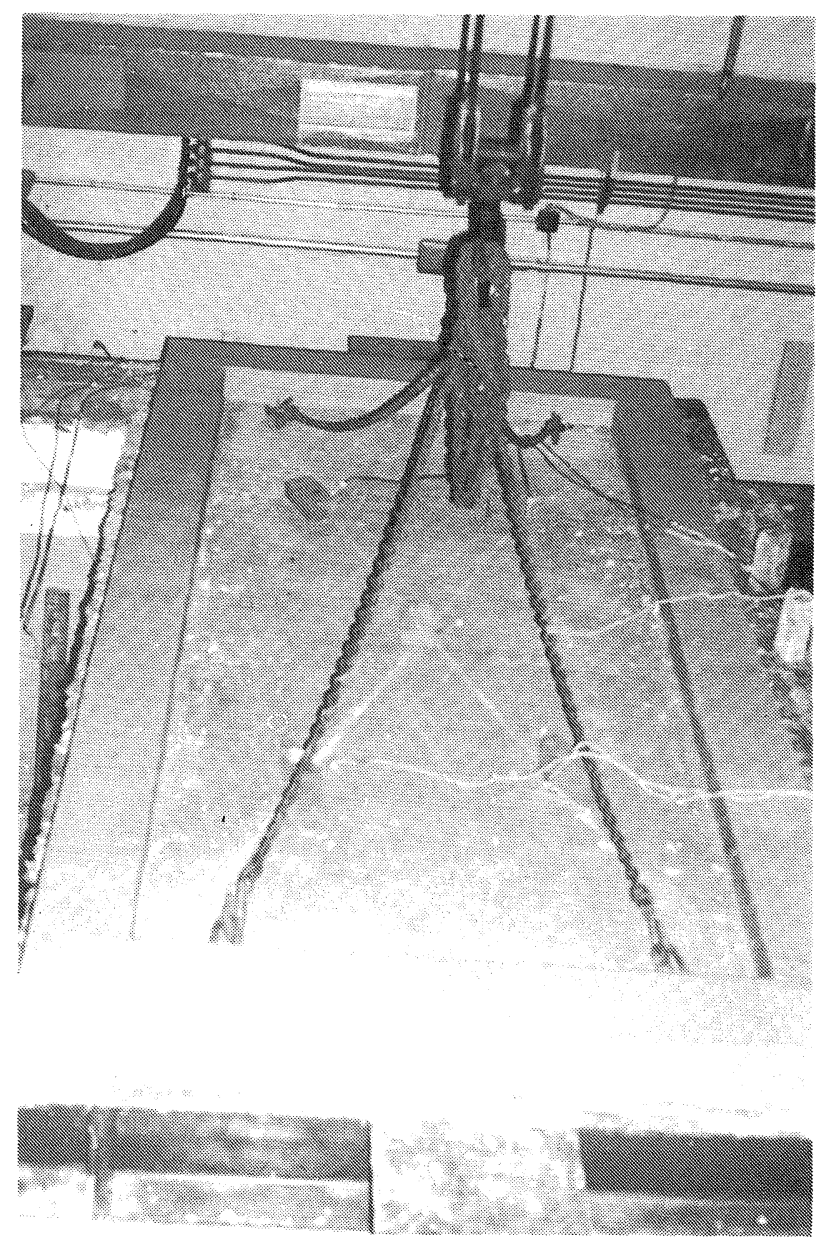

Cubierta de buque con aislamiento, resistente al fuego.

\section{LA INDUSTRIA Y EL USUARIO}

Ante este panorama general, no debe extrañarnos que el usuario o la industria, como hemos dicho, se encuentren confusos a la hora de disponer o proporcionar elementos de calidad frente al fuego. Algunos, por desconocimiento, y otros aprovechando las discontinuidades de la cadena de control de la calidad de los productos ofrecen éstos, o los utilizan sin las debidas garantías.

El efecto de este fenómeno es muy negativo para los avances en el campo del fuego, pues los industriales que colaboran y se interesan por la investigación de sus fabricados tienen que cargar dichos costos al producto final de los mismos, los cuales, de esta manera, a pesar de ofrecer garantías de calidad frente al fuego, al tener mayor costo, se encuentran en desventaja frente a productos incontrolados que se ofrecen en el mercado a precios muy inferiores.

En estas condiciones, sin un control adecuado de la Administración, o sin una exigencia de calidad del usuario, el industrial que ofrece garantías y calidad se encuentra implicitamente penalizado. 
Ya hemos mencionado que en la industria de la madera aquella cadena de control no tiene discontinuidad desde la entrada de la muestra al laboratorio de ensayos, pero tanto en la madera como en los otros materiales, la muestra a ensayar llega al laboratorio generalmente sin control de identificación. Así, por ejemplo, si el Organismo correspondiente precisa comprobar la calidad frente al fuego de una moqueta ignifugada para la apertura de un local (sala de fiestas, bar, etc.) en aplicación de la legislación vigente, debe recoger la muestra de dicho local mediante personal que lo acredite y mandarla o hacerla llegar al laboratorio debidamente identificada, o por el contrario, el dueño del local precisará efectuar la toma de muestra y envío posterior a través de notario. Cualquier otra forma puede favorecer el que el dueño del establecimiento envíe una muestra distinta a la existente en el local y debidamente preparada. El laboratorio ensayará y clasificará aquello que recibe, sin intervención en sus antecedentes ni en su utilización posterior. Por supuesto, no puede ser suficiente, por ejemplo, la presentación de un certificado de la firma ignifugadora, en el que se afirme que se ha ignifugado.

La publicación por el Organismo encargado de homologar de una relación periódica de productos y estructuras homologadas, debidamente identificadas, eliminaría muchos de estos problemas y constituiría un incentivo para los fabricantes y una penalización para los que viesen retirados sus productos en caso de haber cometido falsificación o uso indebido de la homologación. Ello facilitaría a la vez la relación entre Industriausuario, de la cual éste siente necesidad, al no saber qué productos o empresas son las que puede utilizar con garantía. Ello es debido a la falta de grupos, asociaciones de fabricantes, etc., en el campo del fuego, pues en general se trabaja de forma independiente para evitar el conocimiento o copia de procedimientos de innovación frente al fuego.

El conocimiento defectuoso de la utilización que debe darse a los productos homologados o ensayados es también causa frecuente de anomalías en el campo del fuego. No es difícil encontrar, por ejemplo, puertas sin homologar en vías de evacuación o sectores de incendio, incluso con cerraduras de canto, no permitidas por la reglamentación, en edificios de nueva construcción y de gran altura. Firmas instaladoras que probablemente mediante la presentación de un certificado extranjero de una puerta cortafuegos, o nacional, que incluso a veces no corresponde a aquella puerta por haber sido modificada; o simplemente por exhibir dicha puerta una cinta roja en su canto; logran pasar la aceptación del arquitecto para su instalación y el visado de licencia por la Administración.

En los materiales o estructuras que no disponen de sello de calidad frente al fuego, es preciso revisar con detalle las condiciones en que se han efectuado los ensayos, para exigirlas en la puesta en obra, pues una misma estructura ofrece resistencias al fuego muy diferentes según se coloque de una u otra forma.

Debe comprobarse también si la estructura a emplear corresponde exactamente a la ensayada, a través de la descripción del certificado de ensayo. Otras veces los laboratorios efectúan ensayos previos o de investigación, que no se ejecutan según las normas de ensayo para homologación o empleo final (en hornos de menores dimensiones, sin sobrepresión, con temperatura interna diferente a la de la norma, muestras de dimensiones reducidas, etc.) Estos ensayos no pueden aceptarse para estructuras que se llaman homologadas, es decir, para la puesta en obra. Todo ello se anota debidamente en el informe de ensayo. El Arquitecto o el usuario deben comprobar si la estructura que instala o recibe corresponde a ese certificado, el montaje con que se ha ensayado y si el certificado impone alguna restricción.

Anotamos también que no son válidos para nuestro país en cuanto se refiere a los ensayos del fuego los certificados o informes extranjeros. No es cierto, como se ha comprobado, que un ensayo extranjero ofrezca mayores garantías que los nuestros. Por otra parte, al aceptar la NBE-CPI-82 solamente los ensayos efectuados mediante normas UNE no tienen posibilidad de emplear productos que dispongan de certificados extranjeros sin haberse ensayado en nuestro país. Ello podría tener lugar en un futuro, si a través del grupo EGOLF citado se llega a una aceptación entre los laboratorios de dicho grupo de la CEE.

En resumen, todas estas anomalías apuntadas y otras que actualmente existen en el campo del fuego son preocupación de los expertos en este tema. Se está tratando de eliminarlas paulatinamente, pero es lógico que existan en un campo como éste relativamente nuevo. Todas ellas, cuando ocurren catástrofes por incendios, se ponen de manifiesto imposibilitando el esclarecimiento. Sin embargo requieren tratamiento urgente para evitar en lo posible dichas catástrofes. 